\title{
On the Effective Modeling of the Test-Set Non-linearity
}

\author{
Thoalfukar Husseini ${ }^{\star}$, Azam Al-Rawachy ${ }^{\ddagger}$, Syed S. Anera ${ }^{\dagger}$, James Bell $^{\dagger}$, Paul Tasker ${ }^{\dagger}$, Johannes Benedikt $^{\dagger}$ \\ ${ }^{\star}$ Technical Institute of Karbala, Al-Furat Al-Awsat Technical University, Karbala, Iraq \\ ${ }^{\dagger}$ School of Engineering, Cardiff University, Cardiff, UK, CF24 3AA \\ †Engineering Department, Mosul University, Mosul, Iraq \\ Email : Husseinith@Cardiff.ac.uk
}

\begin{abstract}
This paper investigates and compares the use of nonlinear test-set models for an effective and accurate operation of active load-pull systems. The results demonstrate a simpleto-implement and yet robust technique to align the generator and receiver reference plane with a minimum set of required measurements. With only 14 measurements a high agreement between target and measured load points was achieved with an average error less than $0.1 \mathrm{~dB}$ over a $70 \mathrm{~dB}$ dynamic range. An increase in modeling complexity has therefore yielded no improvement. To compare the results, a behavioral model was employed, and it is shown that a high order of model complexity is required to achieve the same level of accuracy. The presented work provides, for the first time, a practical and effective method for the modeling of test-set nonlinearities, hence allowing a costeffective implementation of active load-pull systems that operate power amplifiers within a gain compression region.
\end{abstract}

Index Terms-Automated Active Load-pull, Behavioral model, Generator setting.

\section{INTRODUCTION}

Load-pull measurements are becoming more important in the design and modeling of power amplifier (PA) [1], however the traditional load-pull techniques have shown a shortage in the execution speed due to the number of iterations required to achieve a specific load target [2]. This time-consuming iteration must be minimized to speed up characterization process [3].

A key aspect is the accurate nonlinear modeling of the signaling path between the generator, which controls the injected $a_{2}$ signal. It allows for the use of load-pull amplifiers within their nonlinear regime to keep their required maximum output power at a minimum. This in turn reduces the overall costs of the nonlinear measurement system. It is an aspect of particular relevance for systems operating at high-frequencies where power amplifiers with sufficiently high output power are either not available or come with a high cost-premium.

Several types of strategies have been used to speed up the load-pull measurement and mitigate number of iterations. A typical approach is the use of look-up-tables to correct for the AM-to-AM and AM-to-PM non-linearties of the load-pull amplifiers. However, little has been reported in the literature on its implementation and the achieved accuracy improvements in setting targeted load-impedances within the first iteration. There are number of potential techniques, albeit often more complex in nature, that might be employed to further improve the load-pull performance. One of those approaches is the use of a behavioral model.

This paper investigates new methods to model the PA's nonlinearties. For this purpose, a look-up table (LUT) method is developed which is based on gradients of AM-to-AM and AM-to-PM of the load-pull amplifier (LPA). In addition, the Cardiff model has been reformulated to account for the simplified loading conditions of the LPA and its performance is used as a reference. The investigation also seeks to identify the minimum number of measurements for both approaches that are required to achieve sufficient load-pull accuracy with the first iteration. The objective is to provide a guide for the implementation of new and cost-effective active load pull system.

\section{LOAD-PULl MEASUREMENT SySTEM}

Fig. 1 shows the block diagram of the high-speed nonlinear measurement system that has been utilized to carry out the measurements in this paper. The new systems architecture based on high speed PXIe modules is conceptually similar to previously published systems that are based on a Vector Network Analyzer (VNA) [4]. Data acquisition is performed by vector signal analyzers (VSA). For the signal generation, vector signal generators (VSG) with magnitude and phase control are employed. These are used to provide the stimulus for the device under test (DUT) and the open-loop active loadpull at the fundamental and harmonic frequencies.

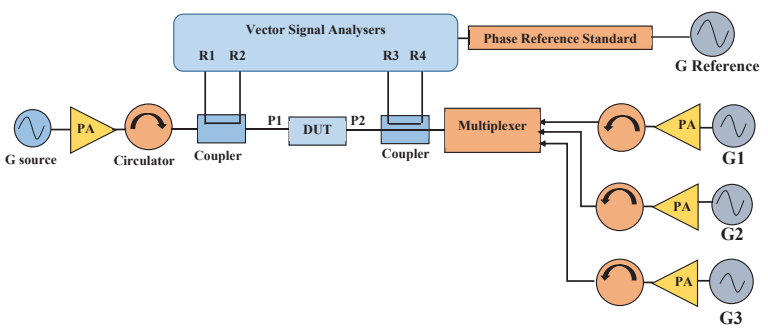

Fig. 1: Block diagram of the high-speed measurement system, which is realized PXIe instrumentation.

The open-loop active load-pull in this work is relying on a Cardiff load-based model to predict the required injected signals (1). It allows for the prediction of the DUT nonlinear response and obtain the correct $a_{2}$ signal for a targeted load impedance with a single iteration.

$$
b_{2}=\sum_{D=0}^{D=1} \sum_{C=-(M-D)}^{C=+(M-D+1)} K_{|C|+2 D, C}\left|\Gamma_{2}\right|^{|C|+2 D}\left(\frac{\Gamma_{2}}{\left|\Gamma_{2}\right|}\right)^{C}
$$


This model structure is a variant of the Cardiff behavioral model. Here, the $b_{2}$ signal is a function of the load reflection coefficient $\Gamma_{2}$. The user defined parameter, M, defines the model complexity, while $K_{p, q}$ are the extracted model coefficients.

The predicted signal $a_{2}$ computed by the Load-based model according to (1) does not take into consideration the effect of the passive and active components which fall between generator and the DUT, such as couplers, circulators, PA drivers and cables. This hinders in achieving load targets during the load-pull measurements, since the phase and magnitude of the injected signal $a_{2}$ are not completely accounted for.

In an effort to overcome this problem, different techniques have been used. In principle, there are two approaches. A numerical technique [5] allows to compensate any differences between the measured and target loads values through use of successive iterations and compensates for the nonlinear DUT and LPA responses. Relatively, simple methods such as Newton-Raphson algorithm provide here a robust and continuously improving approximation at the expense of a large number of iterations. To overcome these issue, a dedicated nonlinear model of the PA path can be employed. This allows for the load-pull amplifiers to operate into their non-linear regime while allowing for the accurate generation of the load impedances.

\section{Measurement Setup and Model Extraction}

The measurement configuration required for extracting nonlinear LPA models is depicted in Fig. 1. This setup should involve all the hardware required for the load-pull measurement to ensure that every loss and amplification is included through the model computation. This measurement requires port 1 and port 2 to be terminated by $50 \mathrm{Ohm}$, as the termination eliminates unwanted signal reflections during the measurement of a-wave signal.

Fundamentally, the non-linear large-signal traveling wave response at the DUT output $\left(a_{2}\right)$ of a PA during this measurement is mathematically related to the generated traveling wave stimulus $a_{L P}$ that is provided the VSAs. The non-linearity of the generated signal is a consequence of the PAs behavior when driven at high power level. The extracted model coefficient must quantify the generic relationship; $a_{2}=f\left(a_{L P}\right)$. The load based-model equation that mentioned in the previous section, was specifically reformulated (2) in order to provide a simplified polynomial equation.

$$
a_{L P}=\left(\frac{a_{2}}{\left|a_{2}\right|}\right) \sum_{n=0}^{n=\infty} K_{2 n+1}\left|a_{2}\right|^{2 n+1}
$$

This equation provides a mathematical framework that can be used to characterize a signal path of the loadpull test-set to move the generator reference plane to the device reference plane. To have a full utilization of the power amplifier that is used within the measurement setup, the model has to be generated while the PA is

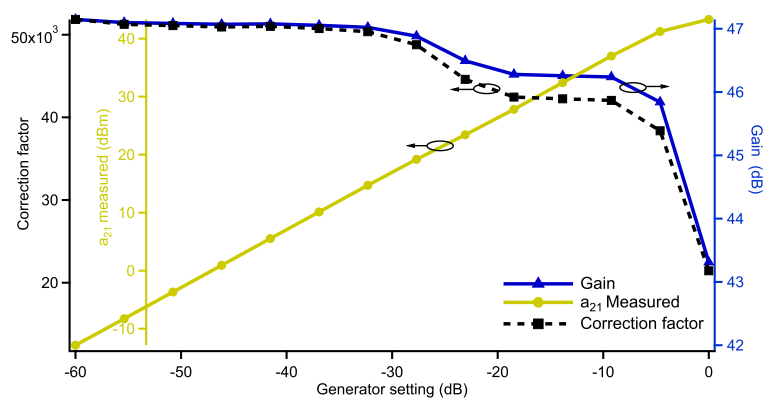

Fig. 2: Generator setting versus $a_{21}$-wave measured, gain and associated correction factors of the PA.

driven into the compression region as shown in Fig 2 .

For the generation of the Cardiff model, an $a_{L P}$ spiral is generated by the VSG and the $a_{2}$ response measured by the receivers (VSAs) within the measurement system. The generated spiral is shown in Fig. 3 and includes both magnitude and phase changes. The magnitude values span over the same dynamic range of the PA performance as shown in Fig. 2. This measurement is used to generate Cardiff models with an increasing complexity.

The error of the generated Cardiff model is estimated from the difference between the actual $a_{L P}$ settings and the values predicted by the models. The overall error is estimated using the minimum normalized mean square error (NMSE) algorithm. Table I shows that sufficient accuracy can be achieved with a model complexity of $13^{\text {th }}$ or higher, i.e. $n=6$ in (2).

TABLE I: NMSE of different model complexity

\begin{tabular}{|c|c|c|c|c|c|c|}
\hline Complexity & $3^{r d}$ & $5^{t h}$ & $7^{t h}$ & $9^{t h}$ & $11^{t h}$ & $13^{t h}$ \\
\hline $\mathrm{n}$ & 1 & 2 & 3 & 4 & 5 & 6 \\
\hline Error (dB) & -25.1 & -29.5 & -34.7 & -39.6 & -44.34 & -47 \\
\hline
\end{tabular}

Fig. 3 shows the high agreement between the generator setting that has been used for the model generation and the generator setting when predicted by the generated model.

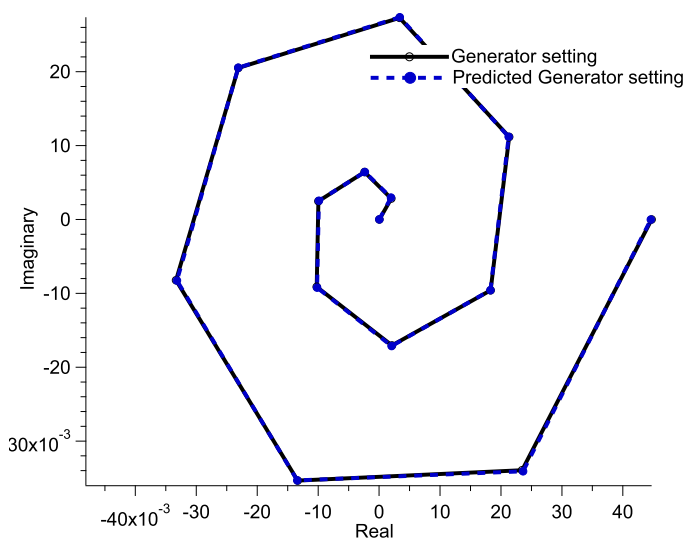

Fig. 3: $13^{\text {th }}$ order Model verification 
The obtained Cardiff model coefficients can be saved in a database and re-used during the forthcoming measurements. This is particularly beneficial for the load-pull measurement because it can be applied directly during the measurement on the required powers $a_{2}$ and then converted for a suitable generator setting.

The second investigated LPA model utilized the same measurement data to generate a look-up table (LUT). As the absolute AM-to-AM and AM-to-PM values of the LPA exhibit a relatively strong variations, a large number of measurement points would be required to represent the nonlinear behavior of the amplifier. To keep the number of required measurements at a minimum the utilized LUT model is based on the gradient of the measured AM-to-AM and AM-to-PM curves. The table is comprised of the indexed $a_{2}$ measured and stored correction factors (CF) for magnitude and phase response of the LPA path where $\mathrm{CFs}$ are calculated according to (3).

$$
C F=\frac{a_{2}}{a_{L P}}
$$

The calculated magnitude CFs shown in Fig. 2 (black trace) and clearly show a dependence on the gain of the LPA path. At the flat gain region the value of the CFs are relatively constant while it drops once the PA operate through the nonlinear region, when its' gain is compressed accordingly.

The look-up table is used to transform the required a-wave at the DUT reference plane to its corresponding generator setting by applying the ratio calculated in (3). For example, if the a-wave at the device reference plane is $a_{2}$ its related correction factor will be looked-up from the stored look-up table. To apply the correction factor, eq. (3) will be rearranged and solved for $a_{L P}$. The implementation utilizes nearest value interpolation, therefore, for power values between the indexed $a_{2}$ data points, the nearest power index is looked-up and the associated magnitude and phase CFs are used to compensate the loss and amplification through the signal path between the load-pull generator and the DUT reference plane.

To experimentally verify the two modeling approaches over a set generator settings, a direct comparison between the target and measured power points is illustrated in Figs. 4 and 5.

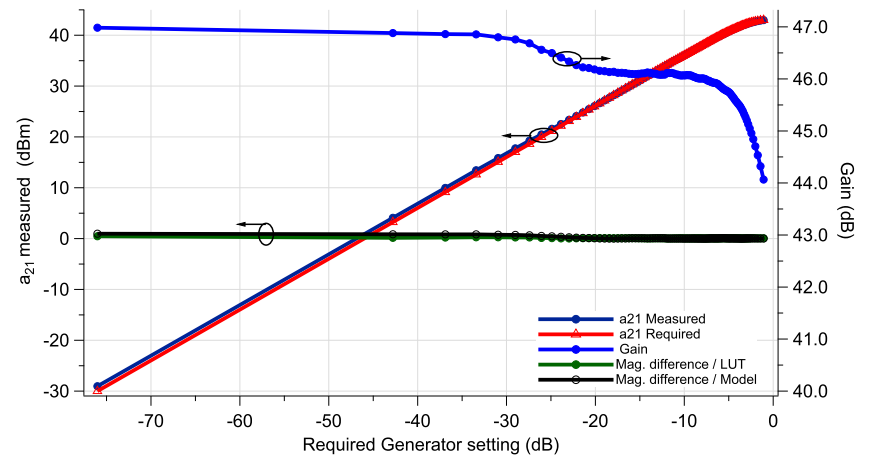

Fig. 4: Comparison between required and measured $a_{21}$ at linear and compression region

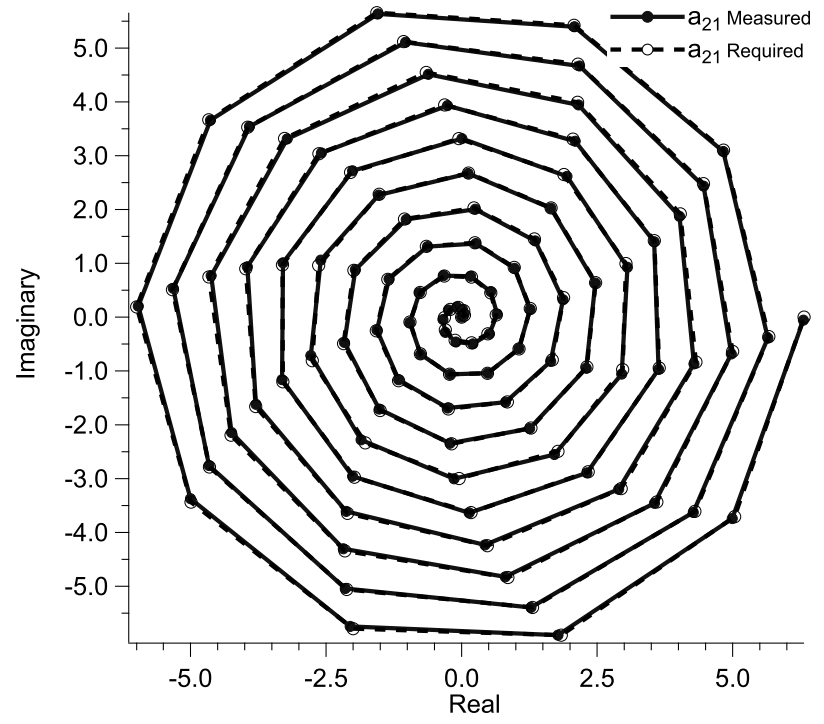

Fig. 5: Illustration of the agreement in phase and magnitude between required and measured $a_{21}$

Fig. 4 shows an excellent agreement is obtained for both type of models over a dynamic range of $75 \mathrm{~dB}$ including the $3 \mathrm{~dB}$ compression region of the PA. Here, a $13^{\text {th }}$ order Cardiff model and an LUT comprising 14 measurements are utilized, i.e. for the generation of the two models the same number of measurement was used. The average residual difference between target and measured powers is $0.1 \mathrm{~dB}$ for the Cardiff model. The residual difference that results when using the LUT, which has been obtained from the same power sweep. The average magnitude residual difference for the same number of measurements is slightly lower at approx. $0.06 \mathrm{~dB}$.

This comparison has been expanded over a range of measurements that is used for the generation of both models. The results are shown in Table II. As it can be seen 14 measurements are sufficient for an accurate $a_{L P}$ prediction. Both models exhibit similar error, approx. $-23 \mathrm{~dB}$, over the increasing number of measurements. In fact, the LUT models shows a slightly better overall performance despite its simplicity.

TABLE II: Comparison between the NMSE of the Cardiff model (C.M) and the look-up table (LUT) approach

\begin{tabular}{|c|c|c|c|c|}
\hline No. meas. & 14 & 21 & 28 & 36 \\
\hline Er./C.M & -23.6 & -23.5 & -23.5 & -23.4 \\
\hline Er./LUT & -23.3 & -23.63 & -23.7 & -23.59 \\
\hline
\end{tabular}

Fig. 6 shows how the generated model has been experimentally used during a load-pull measurement, to predict a generator setting required for emulating specific load targets on the Smith Chart.

It should be noted that to obtain these results a Cardiff model for DUT was employed to predict its non-linear response. The DUT model constantly updates from any in- 


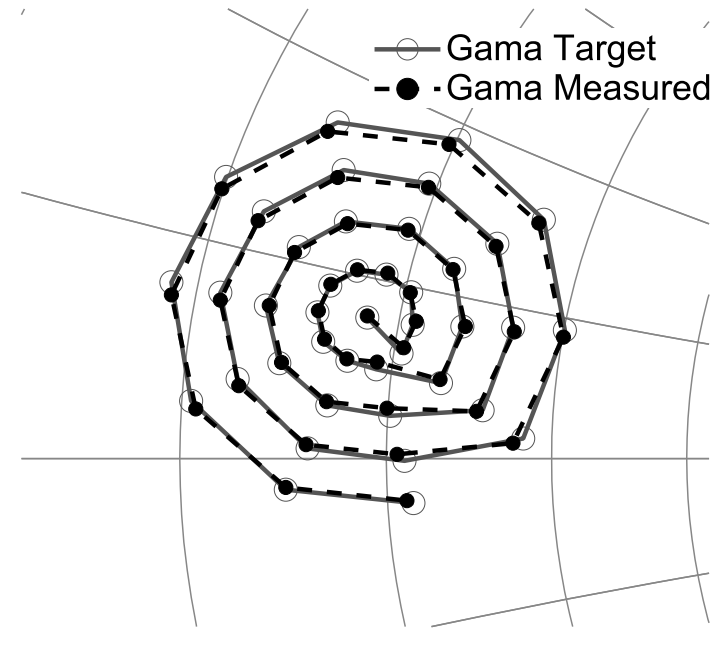

Fig. 6: Comparison between required and measured $a_{21}$ at a DUT reference plane

coming measurement data. The comparison has shown again the high agreement between measured and targeted loads for a load-pull measurement performed on $10 \mathrm{~W}$ Cree device at $1 \mathrm{GHz}$. The excellent results might be ultimately a combination of both the nonlinear DUT and LPA models. Nevertheless, the addition of the nonlinear LPA model provides a significant improvement of the load-pull accuracy. A load-pull measurement utilizes the same sweep as shown in Fig. 6 and using a constant $\mathrm{CF}$ over the entire dynamic range of the signal produced an error of $-16.8 \mathrm{~dB}$

\section{CONCLUSION}

This paper investigated different approached for the nonlinear modeling of the LPA signal path within a load-pull system. These models are used to characterize losses and amplifications of a load-pull test-set that effect a required injected signal at DUT reference plane. The models have been experimentally validated under large-signal excitations and their performance compared. Both methods have shown a very good agreement between measured and targeted powers wherein the average magnitude residual difference for the same number of measurements is $0.1 \mathrm{~dB}$. Moreover, it has been found that 14 measurements are enough to generate sufficiently accurate models with an average error at approx. $23 \mathrm{~dB}$. This is a rather surprising result given the simplicity of the LUT-based model. The investigation demonstrate clearly that increasing the complexity of the nonlinear LPA model offers little to no benefit and suggest a straight forward method to improve the performance of an open-loop active load pull system.

\section{ACKNOWLEDGMENT}

The authors would like to thank the Karbala Technical Institue, Al-Furat Al-Awsat Technical University for supporting and funding this project.

\section{REFERENCES}

[1] J. Bell, R. Saini, S. Woodington, J. Lees, J. Benedikt, S. Cripps, and P. Tasker, "Behavioral model analysis using simultaneous active fundamental load-pull and harmonic source-pull measurements at x-band," in Microwave Symposium Digest (MTT), 2011 IEEE MTT-S International. IEEE, 2011, pp. 1-4.

[2] M. Thorsell and K. Andersson, "Fast multiharmonic active load -pull system with waveform measurement capabilities," IEEE Transactions on Microwave Theory and Techniques, vol. 60, no. 1, pp. 149-157, Jan. 2012.

[3] M. S. Hashmi, A. L. Clarke, S. P. Woodington, J. Lees, J. Benedikt, and P. J. Tasker, "An accurate calibrate-able multiharmonic active load -pull system based on the envelope load -pull concept," IEEE Transactions on Microwave Theory and Techniques, vol. 58, no. 3, pp. 656-664, Mar. 2010.

[4] P. J. Tasker, "Practical waveform engineering," IEEE Microwave Magazine, vol. 10, no. 7, 2009.

[5] R. S. Saini, S. Woodington, J. Lees, J. Benedikt, and P. J. Tasker, "An intelligence driven active loadpull system," in 75th ARFTG Microwave Measurement Conference, May 2010, pp. 1-4. 